\title{
Analytical investigations on formations of hairy neutral reflecting shells in the scalar-Gauss-Bonnet gravity
}

\author{
Yan Peng ${ }^{\mathrm{a}}$ \\ School of Mathematical Sciences, Qufu Normal University, Qufu 273165, Shandong, China
}

Received: 7 February 2020 / Accepted: 21 February 2020 / Published online: 4 March 2020

(C) The Author(s) 2020

\begin{abstract}
We study scalarization of spherically symmetric neutral reflecting shells in the scalar-tensor gravity. We consider neutral static massless scalar fields non-minimally coupled to the Gauss-Bonnet invariant. We obtain a relation representing the existence regime of hairy neutral reflecting shells. For parameters unsatisfying this relation, the massless scalar field cannot exist outside the neutral reflecting shell. In the parameter region where this relation holds, we get analytical solutions of scalar field hairs outside neutral reflecting shells.
\end{abstract}

\section{Introduction}

One well known property of classical black holes is the famous no hair theorem, which states that spherically symmetric black holes cannot support static scalar field hairs in the asymptotically flat background, see references [1-9] and reviews $[10,11]$. The belief in this no hair behavior is partly based on the existence of black hole absorbing horizons. According to some candidate quantum-gravity models, quantum effects may prevent the formation of stable blackhole horizons [12-16]. And horizonless compact objects with reflective boundary conditions have been proposed as alternatives to the familiar (classical) black-hole spacetimes [1723]. So it is interesting to study properties of horizonless reflecting objects.

Interestingly, no hair theorem also holds in such horizonless reflecting object backgrounds. Hod firstly proved that massive static scalar field hairs cannot form in the background of neutral horizonless reflecting objects [24]. This no hair theorem for neutral horizonless reflecting objects was also extended to the case of massless scalar field hairs $[25,26]$. Considering a positive cosmological constant, it was found that the no hair theorem still holds in the background of neutral horizonless reflecting objects [27]. This no hair theo-

a e-mail: yanpengphy@ 163.com (corresponding author) rem for composed system of scalar fields and neutral horizonless reflecting objects was further generalized by including couplings between scalar fields and Ricci curvature [25,28]. However, when horizonless reflecting objects are charged, analytical and numerical results showed that scalar field hairs can exist [29-38]. From front progress, we conclude that no static scalar field hair behavior is a very general property in the background of neutral horizonless reflecting objects.

In other modified gravities, whether static scalar field hairs could exist outside neutral horizonless reflecting objects is a question to be answered. On the other side of black holes, usual ways to introduce scalar hairs are considering stationary scalar fields or adding a confinement to the system [3948]. Recently, a novel approach to trigger black hole scalar hairs was provided by considering non-minimal couplings between scalar fields and the Gauss-Bonnet invariant [4955]. Moreover, it was found that this scalar-Gauss-Bonnet coupling can lead to scalar condensations in various black hole models [56-64]. Inspired by these black hole properties, in the background of neutral reflecting compact stars, we have constructed scalar hairy configurations by including scalar-Gauss-Bonnet couplings with numerical methods [65]. In particular, reflecting shell backgrounds usually allow fully analytical studies, which showed that neutral reflecting shells cannot support static scalar hairs $[29,30]$. As a further step, it is interesting to examine whether scalar fields can condense outside neutral reflecting shells in the model generalized by including scalar-Gauss-Bonnet couplings.

This work is organized as follows. We firstly construct a system with static massless scalar fields outside neutral horizonless reflecting shells in the scalar-Gauss-Bonnet gravity. Then we obtain a relation representing existence regime of hairy shells. For parameters satisfying this relation, we get analytical solutions of scalar field hairs outside neutral reflecting shells. The analytical solutions presented in this paper are valid only in the linearized regime of the scalar fields. At last, we give the main conclusion. 


\section{Scalar condensation behaviors around neutral Dirichlet reflecting shells}

\subsection{A characteristic relation for scalar hairy neutral reflecting shells}

We now write down the model with static massless scalar fields non-minimally coupled to the Gauss-Bonnet invariant. The Lagrangian density of this scalar-tensor gravity is described by [49-55]

$\mathcal{L}=R-\left|\nabla_{\mu} \psi\right|^{2}+f(\psi) \mathcal{R}_{G B}^{2}$,

where $\mathrm{R}$ is the Ricci curvature, $\psi(r)$ is a real scalar field, $f(\psi)$ is the coupling function and $\mathcal{R}_{G B}^{2}$ is the source term. In the linear regime, without generality, we can take the coupling function in the form

$f(\psi)=\eta \psi^{2}$

with $\eta$ describing the coupling strength $[51,52]$. The source term is the Gauss-Bonnet invariant given by

$\mathcal{R}_{G B}^{2}=R_{\mu \nu \rho \sigma} R^{\mu \nu \rho \sigma}-4 R_{\mu \nu} R^{\mu \nu}+R^{2}$.

When neglecting matter fields' backreaction on the metric, the Gauss-Bonnet invariant term is

$\mathcal{R}_{G B}^{2}=\frac{48 M^{2}}{r^{6}}$.

We consider spherically symmetric static neutral spacetimes. The metric ansatz in Schwarzschild coordinates is of the form [52]

$d s^{2}=-g(r) d t^{2}+\frac{d r^{2}}{g(r)}+r^{2}\left(d \theta^{2}+\sin ^{2} \theta d \phi^{2}\right)$,

where the metric function is $g(r)=1-\frac{2 M}{r}$ with $M$ corresponding to the ADM mass. The shell radius is imposed at the radial coordinate $r=r_{s}$. Since we concentrate on the horizonless spacetime, the shell radii satisfy the relation $r_{s}>2 M$. The spherically symmetric angular coordinates are labeled as $\theta$ and $\phi$.

With variation methods, we get the exact linearized scalar equation [49-55]

$\nabla^{\nu} \nabla_{\nu} \psi+\eta \mathcal{R}_{G B}^{2} \psi=0$.

By employing the line element (5), the scalar equation takes the form [65]

$\psi^{\prime \prime}+\left(\frac{2}{r}+\frac{g^{\prime}}{g}\right) \psi^{\prime}+\frac{\eta \mathcal{R}_{G B}^{2}}{g} \psi=0$

with $g=1-\frac{2 M}{r}$ and $\mathcal{R}_{G B}^{2}=\frac{48 M^{2}}{r^{6}}$.

In the limit case of $M \ll r_{s}$, the functions are $g(r)=$ $1-\frac{2 M}{r} \rightarrow 1$ and $g^{\prime}(r)=\frac{2 M}{r^{2}} \rightarrow 0$ as assumed in Refs. $[29,31]$. In the large-r regime, $g^{\prime}(r)$ is neglected and $g(r)$ is set to be 1 . The presence of a coupling parameter $\eta$ is crucial for the existence of a non-trivial analytical solution. With the nonzero term $\eta \mathcal{R}_{G B}^{2}=\frac{48 \eta M^{2}}{r^{6}}, \eta$ appears in the scalar field equation. It means that we study the scalar condensation in the large $\eta$ regime. In this shell background, the Eq. (7) can be expressed as

$\psi^{\prime \prime}+\frac{2}{r} \psi^{\prime}+\frac{48 \eta M^{2}}{r^{6}} \psi=0$.

In order to solve the equation, we need boundary conditions of the scalar field. The asymptotic behavior of the massless scalar field near the infinity boundary is

$$
\psi \propto \frac{1}{r} \text { for } r \rightarrow \infty
$$

So the infinity boundary condition is

$\psi(\infty)=0$.

At the shell radius, we impose Dirichlet reflecting boundary conditions that the scalar field vanishes. So the scalar field condition at the surface is

$\psi\left(r_{s}\right)=0$.

We introduce a new radial function $\tilde{\psi}=\sqrt{r} \psi$. According to $(8), \tilde{\psi}$ satisfies the differential equation

$r^{2} \tilde{\psi}^{\prime \prime}+r \tilde{\psi}^{\prime}+\left(-\frac{1}{4}+\frac{48 \eta M^{2}}{r^{4}}\right) \tilde{\psi}=0$.

With relations (9) and (11), we get boundary conditions

$\tilde{\psi}\left(r_{s}\right)=0, \quad \tilde{\psi}(\infty)=0$.

From boundary conditions (13), one deduces that the function $\tilde{\psi}$ must possess one extremum point $r=r_{\text {peak }}$ in the range $\left(r_{s}, \infty\right)$. At this extremum point, the scalar field satisfies relations [24]

$\left\{\tilde{\psi}^{\prime}=0\right.$ and $\left.\tilde{\psi} \tilde{\psi}^{\prime \prime} \leqslant 0\right\} \quad$ for $\quad r=r_{\text {peak }}$.

Relations (12) and (14) yield the following inequality

$-\frac{1}{4}+\frac{48 \eta M^{2}}{r^{4}} \geqslant 0$ for $r=r_{\text {peak }}$.

This inequality can be transformed into

$\frac{\sqrt{\eta} M}{r^{2}} \geqslant \frac{1}{8 \sqrt{3}}$ for $r=r_{\text {peak }}$.

Considering $r_{s} \leqslant r_{\text {peak }}$, we conclude that scalar hairy shells should satisfy the relation

$\frac{\sqrt{\eta} M}{r_{s}^{2}} \geqslant \frac{1}{8 \sqrt{3}}$ 
Table 1 Radii of Dirichlet reflecting scalar hairy shells

\begin{tabular}{llllll}
\hline$i$ & 1 & 2 & 3 & 4 & 5 \\
\hline$r_{s i}$ & $1.1161 \eta^{\frac{1}{4}} M^{\frac{1}{2}}$ & $0.7658 \eta^{\frac{1}{4}} M^{\frac{1}{2}}$ & $0.6189 \eta^{\frac{1}{4}} M^{\frac{1}{2}}$ & $0.5332 \eta^{\frac{1}{4}} M^{\frac{1}{2}}$ & $0.4755 \eta^{\frac{1}{4}} M^{\frac{1}{2}}$ \\
\hline
\end{tabular}

(a)

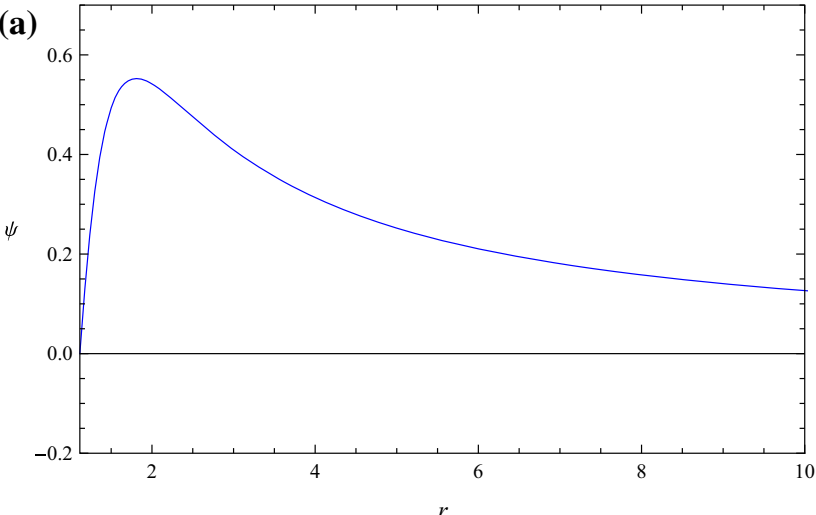

(c)

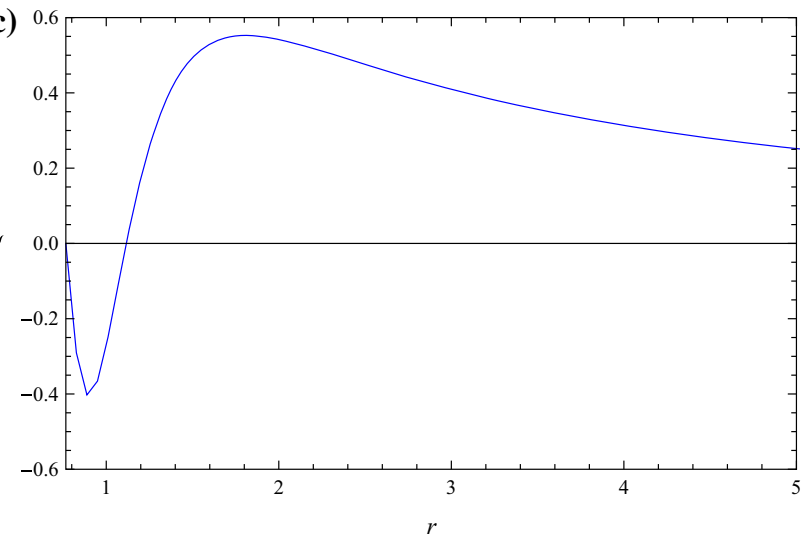

(b)

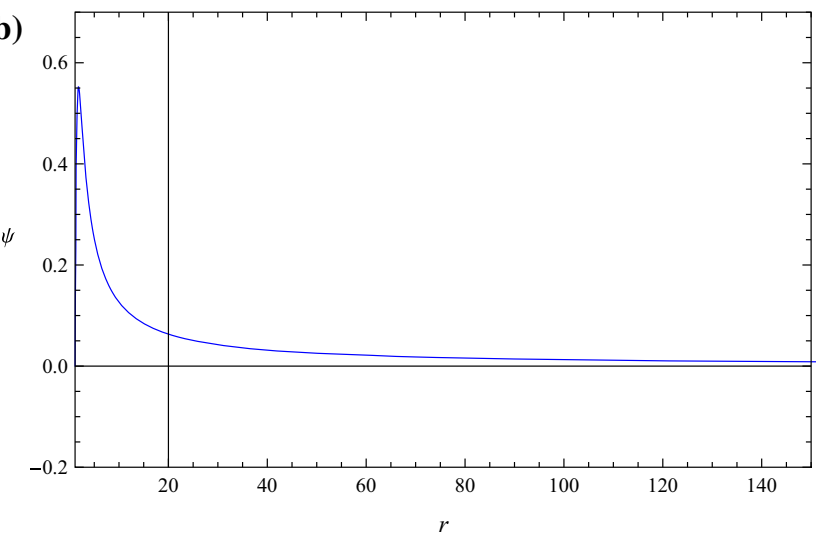

(d)

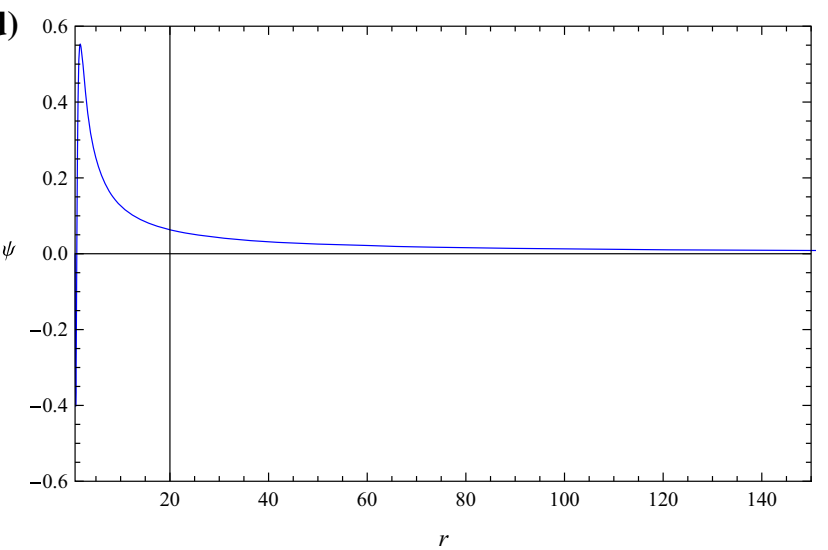

Fig. 1 We plot the scalar field solution around Dirichlet reflecting shells. We take the value $\eta M^{2}=1$ with various $r_{s}$ as: a the case $r_{s 1}=1.1161$ in small region, $\mathbf{b}$ the case $r_{s 1}=1.1161$ in large region, $\mathbf{c}$ the case $r_{s 2}=0.7658$ in small region, $\mathbf{d}$ the case $r_{s 2}=0.7658$ in large region

2.2 Construction of massless scalar field hairy neutral

Dirichlet reflecting shells

In this section, we apply analytical methods to get solutions of scalar field hairs outside Dirichlet reflecting shells in the scalar-Gauss-Bonnet gravity. The general solutions of Eq. (12) can be expressed with Bessel functions in the form [66]

$\tilde{\psi}(r)=A \cdot J_{-\frac{1}{4}}\left(\frac{2 \sqrt{3 \eta} M}{r^{2}}\right)+B \cdot J_{\frac{1}{4}}\left(\frac{2 \sqrt{3 \eta} M}{r^{2}}\right)$

with $A$ and $B$ as integral constants.

At the infinity, the solution (18) asymptotically behaves as

$\tilde{\psi}(r) \propto A \cdot \sqrt{r}+B \cdot \frac{1}{\sqrt{r}}$.

According to the condition (13), the first coefficient $A$ is zero: $A=0$. So the bound-state neutral massless scalar fields are $\psi=\sqrt{\frac{1}{r}} \tilde{\psi}(r)=B \cdot \sqrt{\frac{1}{r}} J_{\frac{1}{4}}\left(\frac{2 \sqrt{3 \eta} M}{r^{2}}\right)$.

With the scalar reflecting condition (11), we get the characteristic scalar field equation

$J_{\frac{1}{4}}\left(\frac{2 \sqrt{3 \eta} M}{r_{s}^{2}}\right)=0$.

If we find parameters satisfying (21), scalar field hairs exist. Defining a new parameter $x=\frac{\sqrt{\eta} M}{r_{s}^{2}}$, there is $x \geqslant \frac{1}{8 \sqrt{3}}$ according to (17). The remaining question is to solve the equation

$J_{\frac{1}{4}}(2 \sqrt{3} x)=0$

in the region $x \geqslant \frac{1}{8 \sqrt{3}}$. With numerical methods, the condition (22) determines discrete values of $x_{i}$

$\cdots>x_{3}>x_{2}>x_{1}=x_{\text {min }} \geqslant \frac{1}{8 \sqrt{3}}$. 
Table 2 Radii of Dirichlet reflecting scalar hairy shells

\begin{tabular}{llllll}
\hline$i$ & 1 & 2 & 3 & 4 & 5 \\
\hline$r_{s i}$ & $1.8090 \eta^{\frac{1}{4}} M^{\frac{1}{2}}$ & $0.8992 \eta^{\frac{1}{4}} M^{\frac{1}{2}}$ & $0.6823 \eta^{\frac{1}{4}} M^{\frac{1}{2}}$ & $0.5720 \eta^{\frac{1}{4}} M^{\frac{1}{2}}$ & $0.5022 \eta^{\frac{1}{4}} M^{\frac{1}{2}}$ \\
\hline
\end{tabular}

(a)

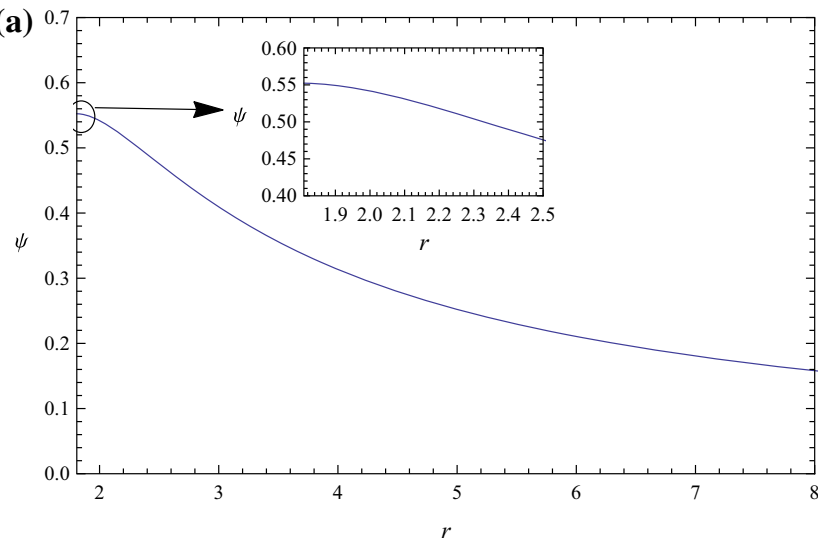

(c)

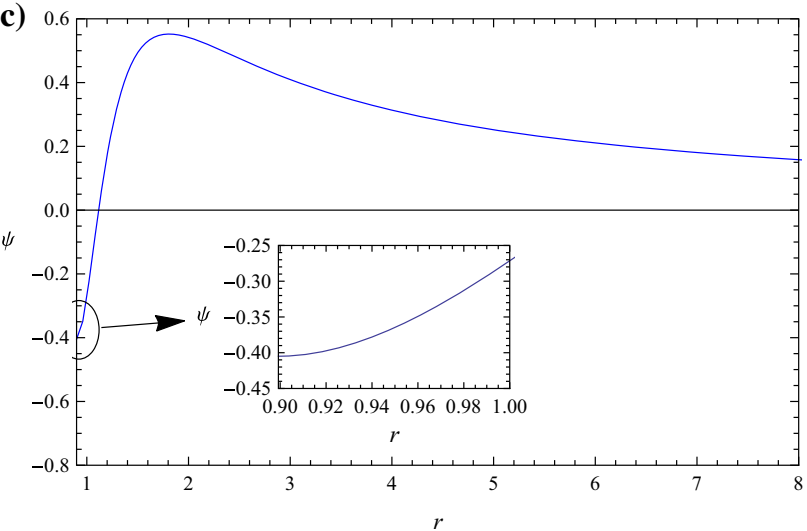

(b)

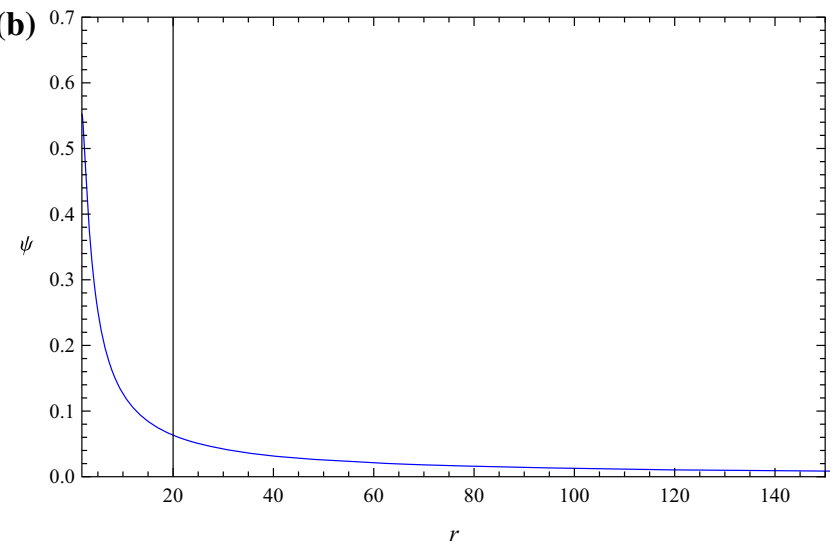

(d)

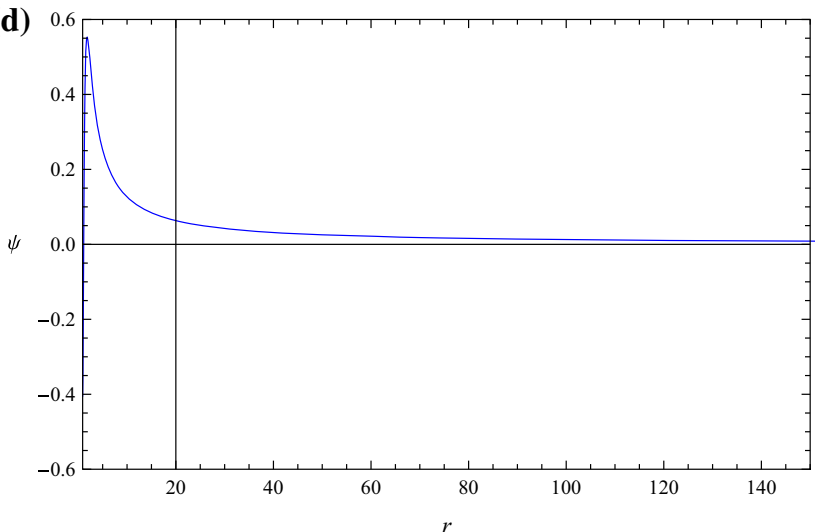

Fig. 2 We show behaviors of the scalar field around Neumann reflecting shells. We take the value $\eta M^{2}=1$ with various $r_{s}$ as: a the case $r_{s 1}=1.8090$ in small region, $\mathbf{b}$ the case $r_{s 1}=1.8090$ in large region, $\mathbf{c}$ the case $r_{s 2}=0.8992$ in small region, $\mathbf{d}$ the case $r_{s 2}=0.8992$ in large region

Fixing shell radii at $r_{s i}=\frac{\eta^{\frac{1}{4}} M^{\frac{1}{2}}}{x_{i}^{\frac{1}{2}}}$, the corresponding scalar field is $\psi \propto \sqrt{\frac{1}{r}} J_{\frac{1}{4}}\left(\frac{2 \sqrt{3 \eta} M}{r^{2}}\right)$ in the form of (20). In Table 1 , we show various values of $r_{s i}$ with respect to $i$. We plot the first two solutions of scalar fields in the background of Dirichlet reflecting shells in Fig. 1. The scalar fields start from zero at the shell radii and asymptotically approach zero at the infinity.

\section{Scalar condensation behaviors around neutral Neumann reflecting shells}

Now we turn to study scalar hair formations in the background of neutral reflecting shells with Neumann surface boundary conditions. At the surface, we impose the Neumann reflecting condition $\psi^{\prime}\left(r_{s}\right)=0$. The derivative of the function $\tilde{\psi}$ satisfies boundary conditions

$$
\begin{aligned}
\tilde{\psi}^{\prime}\left(r_{s}\right) & =\left.(\sqrt{r} \psi)^{\prime}\right|_{r=r_{s}}=\frac{1}{2 \sqrt{r_{s}}} \psi\left(r_{s}\right)+\sqrt{r_{s}} \psi^{\prime}\left(r_{s}\right) \\
& =\frac{1}{2 \sqrt{r_{s}}} \psi\left(r_{s}\right)=\frac{1}{2 r_{s}} \sqrt{r_{s}} \psi\left(r_{s}\right)=\frac{1}{2 r_{s}} \tilde{\psi}\left(r_{s}\right) .
\end{aligned}
$$

The case of $\widetilde{\psi}\left(r_{s}\right)=0$ is just the model studied in Sect. 2 . In this part, we focus on the case of $\widetilde{\psi}\left(r_{s}\right) \neq 0$. In the case of $\tilde{\psi}\left(r_{s}\right)>0$, the function $\tilde{\psi}$ increases to be more positive around the surface and then decreases asymptotically to be zero. In another case of $\widetilde{\psi}\left(r_{s}\right)<0$, the function decreases to be more negative around the surface and then increases to be zero at the infinity. For both cases, one extremum point $r=r_{\text {peak }}$ satisfying (14) exists. Following analysis in part A of Sect. 2, for scalar hairy Neumann reflecting shells, we can easily get the same relation as (17) in the form

$$
\frac{\sqrt{\eta} M}{r_{s}^{2}} \geqslant \frac{1}{8 \sqrt{3}}
$$


With the scalar field solution (20), we can express the Neumann reflecting condition as

$$
\begin{aligned}
\left.\frac{d \psi}{d r}\right|_{r=r_{s}} & =\left.\frac{d}{d r}[\sqrt{r} \tilde{\psi}]\right|_{r=r_{s}} \\
& =\left.\frac{d}{d r}\left[\sqrt{r} J_{\frac{1}{4}}\left(\frac{2 \sqrt{3 \eta} M}{r^{2}}\right)\right]\right|_{r=r_{s}}=0 .
\end{aligned}
$$

The Eq. (26) can be solved through numerical methods. In the parameter regime obeying (25), we obtain discrete values of shell radii which can support the existence of static neutral massless scalar fields. We give the discrete shell radii in Table 2. We also plot the first two solutions of scalar fields outside Neumann reflecting shells in Fig. 2. The scalar fields start with $\psi^{\prime}\left(r_{s}\right)=0$ at the radii and asymptotically approaches zero in the large $r$ region.

\section{Conclusions}

We studied condensations of static massless scalar fields nonminimally coupled to the Gauss-Bonnet invariant outside neutral reflecting shells. At the shell radii, we imposed scalar reflecting boundary conditions. We took two types of reflecting conditions, which are Dirichlet and Neumann reflecting boundary conditions. For both types of conditions, we analytically obtained a characteristic relation for hairy shells in the form $\frac{\sqrt{\eta} M}{r_{s}^{2}} \geqslant \frac{1}{8 \sqrt{3}}$, where $r_{s}$ is the shell radius, $M$ is the shell mass and $\eta$ is the coupling parameter. For parameters unsatisfying this relation, there is no scalar hair theorem. For parameters obeying this relation, we obtained analytical solutions of massless neutral scalar field hairs.

Acknowledgements We would like to thank the anonymous referee for the constructive suggestions to improve the manuscript. This work was supported by the Shandong Provincial Natural Science Foundation of China under Grant no. ZR2018QA008. This work was also supported by a grant from Qufu Normal University of China under Grant no. xkjjc201906.

Data Availability Statement This manuscript has no associated data or the data will not be deposited. [Authors' comment: I would like to emphasize that all relevant physical and mathematical calculations are explicitly presented in this paper.]

Open Access This article is licensed under a Creative Commons Attribution 4.0 International License, which permits use, sharing, adaptation, distribution and reproduction in any medium or format, as long as you give appropriate credit to the original author(s) and the source, provide a link to the Creative Commons licence, and indicate if changes were made. The images or other third party material in this article are included in the article's Creative Commons licence, unless indicated otherwise in a credit line to the material. If material is not included in the article's Creative Commons licence and your intended use is not permitted by statutory regulation or exceeds the permitted use, you will need to obtain permission directly from the copyright holder. To view a copy of this licence, visit http://creativecomm ons.org/licenses/by/4.0/.

Funded by SCOAP ${ }^{3}$.

\section{References}

1. J.D. Bekenstein, Transcendence of the law of baryon-number conservation in black hole physics. Phys. Rev. Lett. 28, 452 (1972)

2. J.E. Chase, Event horizons in static scalar-vacuum space-times. Commun. Math. Phys. 19, 276 (1970)

3. C. Teitelboim, Nonmeasurability of the baryon number of a blackhole. Lett. Nuovo Cimento 3, 326 (1972)

4. R. Ruffini, J.A. Wheeler, Introducing the black hole. Phys. Today 24, 30 (1971)

5. J.D. Bekenstein, Novel "no-scalar-hair" theorem for black holes. Phys. Rev. D 51(12), R6608 (1995)

6. D. Núñez, H. Quevedo, D. Sudarsky, Black holes have no short hair. Phys. Rev. Lett. 76, 571 (1996)

7. S. Hod, Hairy black holes and null circular geodesics. Phys. Rev. D 84, 124030 (2011)

8. Y. Peng, Hair mass bound in the black hole with non-zero cosmological constants. Phys. Rev. D 98, 104041 (2018)

9. Y. Peng, Hair distributions in noncommutative Einstein-BornInfeld black holes. Nucl. Phys. B 941, 1-10 (2019)

10. J.D. Bekenstein, Black hole hair: 25-years after. arXiv:gr-qc/9605059

11. C.A.R. Herdeiro, E. Radu, Asymptotically flat black holes with scalar hair: a review. Int. J. Mod. Phys. D 24(09), 1542014 (2015)

12. P.O. Mazur, E. Mottola, Gravitational condensate stars: an alternative to black holes. arXiv:gr-qc/0109035

13. B.M.H. Cecilia, Chirenti, Luciano Rezzolla How to tell a gravastar from a black hole. Class. Quant. Grav. 24, 4191-4206 (2007)

14. K. Skenderis, M. Taylor, The fuzzball proposal for black holes. Phys. Rep. 467, 117-171 (2008)

15. V. Cardoso, L.C.B. Crispino, C.F.B. Macedo, H. Okawa, Paolo Pani, Light rings as observational evidence for event horizons: long-lived modes, ergoregions and nonlinear instabilities of ultracompact objects. Phys. Rev. D 90(4), 044069 (2014)

16. M. Saravani, N. Afshordi, R.B. Mann, Empty black holes, firewalls, and the origin of Bekenstein-Hawking entropy. Int. J. Mod. Phys. D 23(13), 1443007 (2015)

17. V. Cardoso, P. Pani, Testing the nature of dark compact objects: a status report. Living Rev. Relativ. 22(1), 4 (2019)

18. C. Barceló, R. Carballo-Rubio, L.J. Garay, Gravitational wave echoes from macroscopic quantum gravity effects. JHEP $\mathbf{1 7 0 5}$, 054 (2017)

19. S. Hod, Stationary bound-state scalar configurations supported by rapidly-spinning exotic compact objects. Phys. Lett. B 770, 186 (2017)

20. J. Abedi, H. Dykaar, N. Afshordi, Echoes from the Abyss: tentative evidence for Planck-scale structure at black hole horizons. Phys. Rev. D 96(8), 082004 (2017)

21. B. Holdom, J. Ren, Not quite a black hole. Phys. Rev. D 95(8), 084034 (2017)

22. E. Maggio, P. Pani, V. Ferrari, Exotic compact objects and how to quench their ergoregion instability. Phys. Rev. D 96(10), 104047 (2017)

23. P. Pani, E. Berti, V. Cardoso, Y. Chen, R. Norte, Gravitational wave signatures of the absence of an event horizon. I. Nonradial oscillations of a thin-shell gravastar. Phys. Rev. D 80, 124047 (2009)

24. S. Hod, No-scalar-hair theorem for spherically symmetric reflecting stars. Phys. Rev. D 94, 104073 (2016)

25. S. Hod, No nonminimally coupled massless scalar hair for spherically symmetric neutral reflecting stars. Phys. Rev. D 96, 024019 (2017)

26. Y. Peng, No hair theorem for massless scalar fields outside asymptotically flat horizonless reflecting compact stars. Eur. Phys. J. C 79(10), 850 (2019) 
27. S. Bhattacharjee, S. Sarkar, No-hair theorems for a static and stationary reflecting star. Phys. Rev. D 95, 084027 (2017)

28. S. Hod, No hair for spherically symmetric neutral reflecting stars: nonminimally coupled massive scalar fields. Phys. Lett. B 773, 208-212 (2017)

29. S. Hod, Charged massive scalar field configurations supported by a spherically symmetric charged reflecting shell. Phys. Lett. B 763, 275 (2016)

30. S. Hod, Marginally bound resonances of charged massive scalar fields in the background of a charged reflecting shell. Phys. Lett. B 768, 97-102 (2017)

31. Y. Peng, B. Wang, Y. Liu, Scalar field condensation behaviors around reflecting shells in Anti-de Sitter spacetimes. Eur. Phys. J. C 78(8), 680 (2018)

32. Y. Peng, Scalar field configurations supported by charged compact reflecting stars in a curved spacetime. Phys. Lett. B 780, 144-148 (2018)

33. S. Hod, Charged reflecting stars supporting charged massive scalar field configurations. Eur. Phys. J. C 78, 173 (2017)

34. Y. Peng, Static scalar field condensation in regular asymptotically AdS reflecting star backgrounds. Phys. Lett. B 782, 717-722 (2018)

35. Y. Peng, On instabilities of scalar hairy regular compact reflecting stars. JHEP 10, 185 (2018)

36. Y. Peng, Hair formation in the background of noncommutative reflecting stars. Nucl. Phys. B 938, 143-153 (2019)

37. M. Khodaei, H. Mohseni Sadjadi, No skyrmion hair for stationary spherically symmetric reflecting stars. Phys. Lett. B 797, 134922 (2019)

38. B. Kiczek, M. Rogatko, Ultra-compact spherically symmetric dark matter charged star objects. JCAP 2019(09), 049 (1909)

39. S. Hod, Stationary scalar clouds around rotating black holes. Phys. Rev. D 86, 104026 (2012)

40. C.A.R. Herdeiro, E. Radu, Kerr black holes with scalar hair. Phys. Rev. Lett. 112, 221101 (2014)

41. C.A.R. Herdeiro, J.C. Degollado, H.F. Rúnarsson, Rapid growth of superradiant instabilities for charged black holes in a cavity. Phys. Rev. D 88, 063003 (2013)

42. N. Sanchis-Gual, J.C. Degollado, P.J. Montero, J.A. Font, C. Herdeiro, Explosion and final state of an unstable ReissnerNordström black hole. Phys. Rev. Lett. 116, 141101 (2016)

43. S.R. Dolan, S. Ponglertsakul, E. Winstanley, Stability of black holes in Einstein-charged scalar field theory in a cavity. Phys. Rev. D 92, 124047 (2015)

44. P. Basu, C. Krishnan, P.N.B. Subramanian, Hairy black holes in a box. JHEP 11, 041 (2016)

45. S.A. Hartnoll, C.P. Herzog, G.T. Horowitz, Holographic Superconductors. JHEP 0812, 015 (2008)

46. Y. Peng, Studies of a general flat space/boson star transition model in a box through a language similar to holographic superconductors. JHEP 1707, 042 (2017)

47. Y. Peng, On the thermodynamics of the black hole and hairy black hole transitions in the asymptotically flat spacetime with a box. Eur. Phys. J. C 78(3), 176 (2018)
48. P. Wang, H. Wu, H. Yang, Thermodynamic geometry of AdS black holes and black holes in a cavity. arXiv:1910.07874 [gr-qc]

49. T.P. Sotiriou, S.-Y. Zhou, Black hole hair in generalized scalartensor gravity. Phys. Rev. Lett. 112, 251102 (2014)

50. D.D. Doneva, S.S. Yazadjiev, New Gauss-Bonnet black holes with curvature induced scalarization in the extended scalar-tensor theories. Phys. Rev. Lett. 120, 131103 (2018)

51. H.O. Silva, J. Sakstein, L. Gualtieri, T.P. Sotiriou, E. Berti, Spontaneous scalarization of black holes and compact stars from a GaussBonnet coupling. Phys. Rev. Lett. 120, 131104 (2018)

52. S. Hod, Spontaneous scalarization of Gauss-Bonnet black holes: analytic treatment in the linearized regime. Phys. Rev. D 100, 064039 (2019)

53. G. Antoniou, A. Bakopoulos, P. Kanti, Evasion of no-hair theorems and novel black-hole solutions in Gauss-Bonnet theories. Phys. Rev. Lett. 120, 131102 (2018)

54. C.A.R. Herdeiro, E. Radu, N. Sanchis-Gual, J.A. Font, Spontaneous scalarisation of charged black holes. Phys. Rev. Lett. 121, 101102 (2018)

55. P.V.P. Cunha, C.A.R. Herdeiro, E. Radu, Spontaneously scalarised Kerr black holes. Phys. Rev. Lett. 123, 011101 (2019)

56. Y. Brihaye, C. Herdeiro, E. Radu, The scalarised SchwarzschildNUT spacetime. Phys. Lett. B 788, 295-301 (2019)

57. Y. Brihaye, B. Hartmann, Charged scalar-tensor solitons and black holes with (approximate) Anti-de Sitter asymptotics. JHEP 1901, $142(2019)$

58. A.R. Carlos, Herdeiro, Eugen Radu, Black hole scalarisation from the breakdown of scale-invariance. Phys. Rev. D 99, 084039 (2019)

59. D.D. Doneva, S. Kiorpelidi, P.G. Nedkova, E. Papantonopoulos, S.S. Yazadjiev, Charged Gauss-Bonnet black holes with curvature induced scalarization in the extended scalar-tensor theories. Phys. Rev. D 98, 104056 (2018)

60. H. Motohashi, S. Mukohyama, Shape dependence of spontaneous scalarization. Phys. Rev. D 99, 044030 (2019)

61. M. Minamitsuji, Taishi Ikeda scalarized black holes in the presence of the coupling to Gauss-Bonnet gravity. Phys. Rev. D 99, 044017 (2019)

62. Y.S. Myung, D.C. Zou, Quasinormal modes of scalarized black holes in the Einstein-Maxwell-scalar theory. Phys. Lett. B 790, 400-407 (2019)

63. D.-C. Zou, Y.S. Myung, Scalarized charged black holes with scalar mass term. arXiv:1909.11859 [gr-qc]

64. C.F.B. Macedo, J. Sakstein, E. Berti, L. Gualtieri, H.O. Silva, T.P. Sotiriou, Self-interactions and spontaneous black hole scalarization. Phys. Rev. D 99, 104041 (2019)

65. Y. Peng, Scalarization of compact stars in the scalar-Gauss-Bonnet gravity. JHEP 1912, 064 (2019)

66. M. Abramowitz, I.A. Stegun, Handbook of Mathematical Functions (Dover Publications, New York, 1970) 